\title{
On semilinear biharmonic equations with concave-convex nonlinearities involving weight functions
}

Lu Yang ${ }^{1,2^{*}}$ and Xuan Wang ${ }^{3}$

\section{${ }^{*}$ Correspondence:} yanglu@|zu.edu.cn

${ }^{1}$ School of Mathematics and Statistics, Lanzhou University, Lanzhou, Gansu 730000, P.R. China ${ }^{2}$ Key Laboratory of Applied Mathematics and Complex Systems, Lanzhou University, Lanzhou, Gansu 730000, P.R. China

Full list of author information is available at the end of the article

\begin{abstract}
In this paper, we consider semilinear biharmonic equations with concave-convex nonlinearities involving weight functions, where the concave nonlinear term is $\lambda f(x)|u|^{q-1} u$ and the convex nonlinear term is $h(x)|u|^{p-1} u$ with $\lambda \in \mathbb{R}^{+}$. By use of the Nehari manifold and the direct variational methods, the existence of multiple positive solutions is established as $\lambda \in\left(0, \lambda_{*}\right)$, here the explicit expression of $\lambda_{*}=\lambda_{*}(f, h, p, q, S)$ is provided. MSC: $35 J 35 ; 35 J 40 ; 35 J 65$
\end{abstract}

Keywords: biharmonic equations; concave-convex nonlinearities; weight functions

\section{Introduction}

In recent years, there has been extensive attention on semilinear second-order elliptic equations,

$$
\begin{cases}-\Delta u=g_{\lambda}(x, u), & \text { in } \Omega, \\ u=0, & \text { on } \partial \Omega,\end{cases}
$$

here $\Omega$ is a bounded smooth domain in $\mathbb{R}^{N}(N \geq 3), g_{\lambda}: \Omega \times \mathbb{R} \rightarrow \mathbb{R}$ and $\lambda$ is a positive parameter; see [1-8] and the references therein. As $g_{\lambda}$ is sublinear, say, $g_{\lambda}=\lambda u^{q}, 0<q<1$, the monotone iteration scheme or the method of sub-solutions and super-solutions are effective; see [9]. As $g_{\lambda}$ is superlinear, for example, $g_{\lambda}=\lambda u+|u|^{p-1} u, 1<p<\frac{N+2}{N-2}$, variational methods are applicable; see [10]. In contrast with the pure sublinear case and the pure superlinear case, in [2] Ambrosetti et al. considered problem (1.1) when $g_{\lambda}$ is, roughly, the sum of a sublinear and a superlinear term. To be precise, they considered the following problem:

$$
\left\{\begin{array}{l}
-\Delta u=\lambda u^{q}+u^{p}, \quad \text { in } \Omega \\
0 \leq u \in H_{0}^{1}(\Omega)
\end{array}\right.
$$

with $0<q<1<p \leq \frac{N+2}{N-2}$. They proved that problem (1.2) admits at least two positive solutions for $\lambda$ sufficiently small. In [6], Sun and Li considered a similar problem:

$$
\left\{\begin{array}{l}
-\Delta u=u^{q}+\lambda u^{p}, \quad \text { in } \Omega, \\
0 \leq u \in H_{0}^{1}(\Omega),
\end{array}\right.
$$

(02014 Yang and Wang; licensee Springer. This is an Open Access article distributed under the terms of the Creative Commons Attribution License (http://creativecommons.org/licenses/by/2.0), which permits unrestricted use, distribution, and reproduction in any medium, provided the original work is properly cited. 
with $0<q<1<p=\frac{N+2}{N-2}$, the authors studied the value of $\Lambda$, the supremum of the set $\lambda$, related to the existence and multiplicity of positive solutions and established uniform lower bounds for $\Lambda$. In [8], Wu considered the subcritical case of problem (1.2) with $\lambda u^{q}$ replaced by $\lambda f(x) u^{q}$, here $f(x) \in C(\bar{\Omega})$ is a sign-changing function, and he showed that problem (1.2) has at least two positive solutions as $\lambda$ is small enough.

Some interesting generalizations of (1.2) have been provided in the framework of quasilinear elliptic equations or systems, semilinear second-order elliptic systems or fourthorder elliptic equations. More recently, the semilinear fourth-order elliptic equations have been studied by many authors, we refer the reader to [11-13] and the references therein. Motivated by some work in $[6,8,13]$, we deal with the following semilinear biharmonic elliptic equation:

$$
\begin{cases}\Delta^{2} u=\lambda f(x)|u|^{q-1} u+h(x)|u|^{p-1} u, & \text { in } \Omega, \\ u=\Delta u=0, & \text { on } \partial \Omega\end{cases}
$$

where $\Omega$ is a bounded smooth domain in $\mathbb{R}^{N}(N \geq 4), 0<q<1<p<2^{* *}\left(2^{* *}=\frac{N+4}{N-4}\right.$ for $N>4$ and $2^{* *}=\infty$ for $\left.N=4\right), \lambda>0$ is a parameter, $f \in C(\bar{\Omega})$ is a positive or sign-changing weight function and $h \in C(\bar{\Omega})$ is a positive weight function.

For convenience and simplicity, we introduce some notations. The norm of $u$ in $L^{r}(\Omega)$ is denoted by $|u|_{r}=\left(\int_{\Omega}|u(x)|^{r}\right)^{1 / r}$, the norm of $u$ in $C(\bar{\Omega})$ is denoted by $|u|_{\infty}=\max _{x \in \bar{\Omega}}|u(x)|$; $H_{0}^{1}(\Omega) \cap H^{2}(\Omega)$ is denoted by $H(\Omega)$, endowed with the norm $\|u\|=|\Delta u|_{2} ; S$ denotes the best Sobolev constant for the embedding of $H(\Omega)$ in $L^{p+1}(\Omega)$ (see [14]); to be precise, $|u|_{p+1} \leq S\|u\|$ for all $u \in H(\Omega)$.

Now we define

$$
J_{\lambda}(u)=\frac{1}{2}\|u\|^{2}-\frac{\lambda}{q+1} \int_{\Omega} f(x)|u|^{q+1} \mathrm{~d} x-\frac{1}{p+1} \int_{\Omega} h(x)|u|^{p+1} \mathrm{~d} x, \quad u \in H(\Omega) .
$$

It is well known that the weak solutions of problem (1.3) are the critical points of the energy functional $J_{\lambda}$ (see Rabinowitz [15]).

Next, we consider the Nehari minimization problem: for $\lambda>0$,

$$
\alpha_{\lambda}(\Omega)=\inf \left\{J_{\lambda}(u) \mid u \in M_{\lambda}(\Omega)\right\},
$$

where $M_{\lambda}(\Omega)=\left\{u \in H(\Omega) \backslash\{0\} \mid\left\langle J_{\lambda}^{\prime}(u), u\right\rangle=0\right\}$. Define

$$
\psi_{\lambda}(u)=\left\langle J_{\lambda}^{\prime}(u), u\right\rangle=\|u\|^{2}-\lambda \int_{\Omega} f(x)|u|^{q+1} \mathrm{~d} x-\int_{\Omega} h(x)|u|^{p+1} \mathrm{~d} x .
$$

Then for $u \in M_{\lambda}(\Omega)$,

$$
\left\langle\psi_{\lambda}^{\prime}(u), u\right\rangle=2\|u\|^{2}-\lambda(q+1) \int_{\Omega} f(x)|u|^{q+1} \mathrm{~d} x-(p+1) \int_{\Omega} h(x)|u|^{p+1} \mathrm{~d} x .
$$

Similarly to the method used in Tarantello [16], we split $M_{\lambda}(\Omega)$ into three parts:

$$
\begin{aligned}
& M_{\lambda}^{+}(\Omega)=\left\{u \in M_{\lambda}(\Omega) \mid\left\langle\psi_{\lambda}^{\prime}(u), u\right\rangle>0\right\}, \\
& M_{\lambda}^{0}(\Omega)=\left\{u \in M_{\lambda}(\Omega) \mid\left\langle\psi_{\lambda}^{\prime}(u), u\right\rangle=0\right\}, \\
& M_{\lambda}^{-}(\Omega)=\left\{u \in M_{\lambda}(\Omega) \mid\left\langle\psi_{\lambda}^{\prime}(u), u\right\rangle<0\right\} .
\end{aligned}
$$


Note that all solutions of (1.3) are clearly in the Nehari manifold, $M_{\lambda}(\Omega)$. Hence, our approach to solve problem (1.3) is to analyze the structure of $M_{\lambda}(\Omega)$, and then to deal with the minimization problems for $J_{\lambda}$ on $M_{\lambda}^{+}(\Omega)$ and $M_{\lambda}^{-}(\Omega)$ applying the direct variational method.

The following is our main result.

Theorem 1.1 Let $\lambda_{*}=\frac{p-1}{p-q} \cdot\left[\frac{1-q}{(p-q)|h|_{\infty}}\right]^{\frac{1-q}{p-1}} S^{\frac{2(p-q)}{1-p}}|f|_{p^{*}}^{-1}$ with $p^{*}=\frac{p+1}{p-q}$, then problem (1.3) has at least two positive solutions for any $\lambda \in\left(0, \lambda_{*}\right)$.

The paper is organized as follows: in Section 2, we give some lemmas; in Section 3, we prove Theorem 1.1.

\section{Preliminaries}

In this section, we prove several lemmas.

Lemma 2.1 For $\lambda \in\left(0, \lambda_{*}\right)$ (where $\lambda_{*}$ is given in Theorem 1.1), we have $M_{\lambda}^{0}(\Omega)=\phi$.

Proof Suppose that $M_{\lambda}^{0}(\Omega) \neq \phi$ for all $\lambda>0$. If $u \in M_{\lambda}^{0}(\Omega)$, then we have

$$
\|u\|^{2}=\lambda \int_{\Omega} f(x)|u|^{q+1} \mathrm{~d} x+\int_{\Omega} h(x)|u|^{p+1} \mathrm{~d} x
$$

and

$$
2\|u\|^{2}=\lambda(q+1) \int_{\Omega} f(x)|u|^{q+1} \mathrm{~d} x+(p+1) \int_{\Omega} h(x)|u|^{p+1} \mathrm{~d} x .
$$

By (2.1)-(2.2), the Sobolev inequality, and the Hölder inequality, we get

$$
\|u\|^{2}=\frac{p-q}{1-q} \int_{\Omega} h(x)|u|^{p+1} \mathrm{~d} x \leq \frac{p-q}{1-q}|h|_{\infty} S^{p+1}\|u\|^{p+1}
$$

and

$$
\|u\|^{2}=\lambda \cdot \frac{p-q}{p-1} \int_{\Omega} f(x)|u|^{q+1} \mathrm{~d} x \leq \lambda \cdot \frac{p-q}{p-1}|f|_{p^{*}} S^{q+1}\|u\|^{q+1}
$$

where $p^{*}=\frac{p+1}{p-q}$. Thus, using (2.3) and (2.4), we have

$$
\begin{aligned}
\lambda & \geq \frac{p-1}{p-q} \cdot|f|_{p^{*}}^{-1} S^{-(q+1)}\left[\frac{1-q}{p-q}|h|_{\infty}^{-1} S^{-(p+1)}\right]^{\frac{1-q}{p-1}} \\
& =\frac{p-1}{p-q} \cdot\left[\frac{1-q}{(p-q)|h|_{\infty}}\right]^{\frac{1-q}{p-1}} S^{\frac{2(p-q)}{1-p}}|f|_{p^{*}}^{-1}=\lambda_{*} .
\end{aligned}
$$

Hence, by (2.5) the desired conclusion yields.

Lemma 2.2 If $u \in M_{\lambda}^{-}(\Omega)$, then

$$
\|u\|>S^{\frac{1+p}{1-p}}\left[\frac{1-q}{(p-q)|h|_{\infty}}\right]^{\frac{1}{p-1}} \text { and } \int_{\Omega} h(x)|u|^{p+1} \mathrm{~d} x>|h|_{\infty}^{\frac{2}{1-p}}\left[\frac{(p-q) S^{2}}{1-q}\right]^{\frac{1+p}{1-p}} \text {. }
$$


Proof From $u \in M_{\lambda}^{-}(\Omega)$, it is easy to see that

$$
\|u\|^{2}<\frac{p-q}{1-q} \int_{\Omega} h(x)|u|^{p+1} \mathrm{~d} x .
$$

By the Sobolev inequality, we get

$$
\|u\|>S^{1+p}\left[\frac{1-q}{(p-q)|h|_{\infty}}\right]^{\frac{1}{p-1}}
$$

In addition,

$$
\int_{\Omega} h(x)|u|^{p+1} \mathrm{~d} x>|h|_{\infty}^{\frac{2}{1-p}}\left[\frac{(p-q) S^{2}}{1-q}\right]^{\frac{1+p}{1-p}}
$$

The proof is completed.

By Lemma 2.1, for $\lambda \in\left(0, \lambda_{*}\right)$ we write $M_{\lambda}(\Omega)=M_{\lambda}^{+}(\Omega) \cup M_{\lambda}^{-}(\Omega)$ and define

$$
\alpha_{\lambda}^{+}(\Omega)=\inf _{u \in M_{\lambda}^{+}(\Omega)} J_{\lambda}(u), \quad \alpha_{\lambda}^{-}(\Omega)=\inf _{u \in M_{\lambda}^{-}(\Omega)} J_{\lambda}(u) .
$$

The following lemma shows that the minimizers on $M_{\lambda}(\Omega)$ are 'usually' critical points for $J_{\lambda}$.

Lemma 2.3 For $\lambda \in\left(0, \lambda_{*}\right)$, if $u_{0}$ is a local minimizer for $J_{\lambda}$ on $M_{\lambda}(\Omega)$, then $J_{\lambda}^{\prime}\left(u_{0}\right)=0$ in $[H(\Omega)]^{*}$.

Proof If $u_{0}$ is a local minimizer for $J_{\lambda}$ on $M_{\lambda}(\Omega)$, then $u_{0}$ is a solution of the optimization problem

$$
\text { minimize } J_{\lambda}(u) \text { subject to } \psi_{\lambda}(u)=0 \text {. }
$$

Hence, by the theory of Lagrange multipliers, there exists $\theta \in \mathbb{R}$ such that

$$
J_{\lambda}^{\prime}\left(u_{0}\right)=\theta \psi_{\lambda}^{\prime}\left(u_{0}\right) \quad \text { in }[H(\Omega)]^{*}
$$

Thus,

$$
\left\langle J_{\lambda}^{\prime}\left(u_{0}\right), u_{0}\right\rangle=\theta\left\langle\psi_{\lambda}^{\prime}\left(u_{0}\right), u_{0}\right\rangle
$$

From $u_{0} \in M_{\lambda}(\Omega)$ and Lemma 2.1, we have $\left\langle J_{\lambda}^{\prime}\left(u_{0}\right), u_{0}\right\rangle=0$ and $\left\langle\psi_{\lambda}^{\prime}\left(u_{0}\right), u_{0}\right\rangle \neq 0$. So, by (2.6)-(2.7) we get $J_{\lambda}^{\prime}\left(u_{0}\right)=0$ in $[H(\Omega)]^{*}$.

For each $u \in H(\Omega) \backslash\{0\}$, we write

$$
t_{\max }=\left(\frac{(1-q)\|u\|^{2}}{(p-q) \int_{\Omega} h(x)|u|^{p+1} \mathrm{~d} x}\right)^{\frac{1}{p-1}}>0 .
$$

Then we have the following lemma. 
Lemma 2.4 For each $u \in H(\Omega) \backslash\{0\}$ and $\lambda \in\left(0, \lambda_{*}\right)$, we have

(i) there is a unique $t^{-}=t^{-}(u)>t_{\max }>0$ such that $t^{-}(u) u \in M_{\lambda}^{-}(\Omega)$ and $J_{\lambda}\left(t^{-}(u) u\right)=\max _{t \geq 0} J_{\lambda}(t u)$

(ii) $t^{-}(u)$ is a continuous function for nonzero $u$;

(iii) $M_{\lambda}^{-}(\Omega)=\left\{u \in H(\Omega) \backslash\{0\} \mid \frac{1}{\|u\|} t^{-}\left(\frac{u}{\|u\|}\right)=1\right\}$;

(iv) if $\int_{\Omega} f(x)|u|^{q+1} \mathrm{~d} x>0$, then there is a unique $0<t^{+}=t^{+}(u)<t_{\max }$ such that $t^{+}(u) u \in M_{\lambda}^{+}(\Omega)$ and $J_{\lambda}\left(t^{+}(u) u\right)=\min _{0 \leq t \leq t^{-}} J_{\lambda}(t u)$.

Proof (i) Fix $u \in H(\Omega) \backslash\{0\}$. Let

$$
s(t)=t^{1-q}\|u\|^{2}-t^{p-q} \int_{\Omega} h(x)|u|^{p+1} \mathrm{~d} x, \quad t \geq 0
$$

Then we have $s(0)=0, s(t) \rightarrow-\infty$ as $t \rightarrow \infty, s(t)$ is concave and reaches its maximum at $t_{\max }$. Moreover,

$$
\begin{aligned}
s\left(t_{\max }\right) & =t_{\max }^{1-q}\|u\|^{2}-t_{\max }^{p-q} \int_{\Omega} h(x)|u|^{p+1} \mathrm{~d} x \\
& =\|u\|^{q+1}\left[\left(\frac{1-q}{p-q}\right)^{\frac{1-q}{p-1}}-\left(\frac{1-q}{p-q}\right)^{\frac{p-q}{p-1}}\right]\left(\frac{\|u\|^{p+1}}{\int_{\Omega} h(x)|u|^{p+1} \mathrm{~d} x}\right)^{\frac{1-q}{p-1}} \\
& \geq\|u\|^{q+1}\left(\frac{p-1}{p-q}\right)\left(\frac{1-q}{p-q}\right)^{\frac{1-q}{p-1}}\left(\frac{1}{|h|_{\infty} S^{p+1}}\right)^{\frac{1-q}{p-1}} .
\end{aligned}
$$

Case I. $\int_{\Omega} f(x)|u|^{q+1} \mathrm{~d} x \leq 0$.

There is a unique $t^{-}>t_{\max }$ such that $s\left(t^{-}\right)=\lambda \int_{\Omega} f(x)|u|^{q+1} \mathrm{~d} x$ and $s^{\prime}\left(t^{-}\right)<0$. Now,

$$
\begin{aligned}
\left\langle J_{\lambda}^{\prime}\left(t^{-} u\right), t^{-} u\right\rangle & =\left\|t^{-} u\right\|^{2}-\lambda \int_{\Omega} f(x)\left|t^{-} u\right|^{q+1} \mathrm{~d} x-\int_{\Omega} h(x)\left|t^{-} u\right|^{p+1} \mathrm{~d} x \\
& =\left(t^{-}\right)^{q+1}\left[s\left(t^{-}\right)-\lambda \int_{\Omega} f(x)|u|^{q+1} \mathrm{~d} x\right] \\
& =0
\end{aligned}
$$

and

$$
\begin{aligned}
\left\langle\psi_{\lambda}^{\prime}\left(t^{-} u\right), t^{-} u\right\rangle & =(1-q)\left\|t^{-} u\right\|^{2}-(p-q) \int_{\Omega} h(x)\left|t^{-} u\right|^{p+1} \mathrm{~d} x \\
& =\left(t^{-}\right)^{2+q}\left[(1-q)\left(t^{-}\right)^{-q}\|u\|^{2}-(p-q)\left(t^{-}\right)^{p-q-1} \int_{\Omega} h(x)|u|^{p+1} \mathrm{~d} x\right] \\
& =\left(t^{-}\right)^{2+q} s^{\prime}\left(t^{-}\right)<0 .
\end{aligned}
$$

Thus, $t^{-} u \in M_{\lambda}^{-}(\Omega)$. In addition,

$$
\begin{aligned}
\frac{\mathrm{d} J_{\lambda}(t u)}{\mathrm{d} t} & =t\|u\|^{2}-\lambda t^{q} \int_{\Omega} f(x)|u|^{q+1} \mathrm{~d} x-t^{p} \int_{\Omega} h(x)|u|^{p+1} \mathrm{~d} x \\
& =t^{-1}\left\langle J_{\lambda}^{\prime}(t u), t u\right\rangle=0 \quad \text { if and only if } \quad t=t^{-}
\end{aligned}
$$


and

$$
\begin{aligned}
\left.\frac{\mathrm{d}^{2} J_{\lambda}(t u)}{\mathrm{d} t^{2}}\right|_{t=t^{-}} & =\|u\|^{2}-\lambda q\left(t^{-}\right)^{q-1} \int_{\Omega} f(x)|u|^{q+1} \mathrm{~d} x-p\left(t^{-}\right)^{p-1} \int_{\Omega} h(x)|u|^{p+1} \mathrm{~d} x \\
& =\left(t^{-}\right)^{-2}\left\langle\psi_{\lambda}^{\prime}\left(t^{-} u\right), t^{-} u\right\rangle<0 .
\end{aligned}
$$

Hence, $J_{\lambda}\left(t^{-} u\right)=\max _{t \geq 0} J_{\lambda}(t u)$.

Case II. $\int_{\Omega} f(x)|u|^{q+1} \mathrm{~d} x>0$.

From (2.8) and

$$
\begin{aligned}
s(0) & =0<\lambda \int_{\Omega} f(x)|u|^{q+1} \mathrm{~d} x \leq \lambda|f|_{p^{*}} S^{q+1}\|u\|^{q+1} \\
& <\|u\|^{q+1}\left(\frac{p-1}{p-q}\right)\left(\frac{1-q}{p-q}\right)^{\frac{1-q}{p-1}}\left(\frac{1}{|h|_{\infty} S^{p+1}}\right)^{\frac{1-q}{p-1}} \\
& \leq s\left(t_{\max }\right) \quad \text { for } \lambda \in\left(0, \lambda_{*}\right),
\end{aligned}
$$

there exist unique $t^{+}$and $t^{-}$such that $0<t^{+}<t_{\max }<t^{-}$,

$$
s\left(t^{+}\right)=\lambda \int_{\Omega} f(x)|u|^{q+1} \mathrm{~d} x=s\left(t^{-}\right)
$$

and

$$
s^{\prime}\left(t^{+}\right)>0>s^{\prime}\left(t^{-}\right)
$$

Similar to the argument in Case I above, we have $t^{+} u \in M_{\lambda}^{+}(\Omega), t^{-} u \in M_{\lambda}^{-}(\Omega)$, and

$$
J_{\lambda}\left(t^{-} u\right)=\max _{t \geq 0} J_{\lambda}(t u), \quad J_{\lambda}\left(t^{+} u\right)=\min _{0 \leq t \leq t^{-}} J_{\lambda}(t u) .
$$

(ii) By the uniqueness of $t^{-}(u)$ and the external property of $t^{-}(u)$, we find that $t^{-}(u)$ is continuous function of $u \neq 0$.

(iii) For $u \in M_{\lambda}^{-}(\Omega)$, let $v=\frac{u}{\|u\|}$. By item (i), there is a unique $t^{-}(v)>0$ such that $t^{-}(v) v \in$ $M_{\lambda}^{-}(\Omega)$, that is, $t^{-}\left(\frac{u}{\|u\|}\right) \frac{1}{\|u\|} u \in M_{\lambda}^{-}(\Omega)$. Since $u \in M_{\lambda}^{-}(\Omega)$, we have $t^{-}\left(\frac{u}{\|u\|}\right) \frac{1}{\|u\|}=1$, which implies

$$
M_{\lambda}^{-}(\Omega) \subset\left\{u \in H(\Omega) \backslash\{0\} \mid \frac{1}{\|u\|} t^{-}\left(\frac{u}{\|u\|}\right)=1\right\} .
$$

Conversely, let $u \in H(\Omega) \backslash\{0\}$ such that $\frac{1}{\|u\|} t^{-}\left(\frac{u}{\|u\|}\right)=1$. Then $t^{-}\left(\frac{u}{\|u\|}\right) \frac{u}{\|u\|} \in M_{\lambda}^{-}(\Omega)$. Therefore,

$$
M_{\lambda}^{-}(\Omega)=\left\{u \in H(\Omega) \backslash\{0\} \mid \frac{1}{\|u\|} t^{-}\left(\frac{u}{\|u\|}\right)=1\right\} .
$$

(iv) By Case II of item (i).

By $f \in C(\bar{\Omega})$ and changes sign in $\Omega$, we have $\Theta=\{x \in \Omega \mid f(x)>0\}$ is an open set in $R^{N}$. Without loss of generality, we may assume that $\Theta$ is a domain in $R^{N}$. Consider the 
following biharmonic equation:

$$
\begin{cases}\Delta^{2} u=h(x)|u|^{p-1} u, & \text { in } \Theta, \\ u=\Delta u=0, & \text { on } \partial \Theta .\end{cases}
$$

Associated with (2.9), we consider the energy functional

$$
K(u)=\frac{1}{2}\|u\|^{2}-\frac{1}{p+1} \int_{\Theta} h(x)|u|^{p+1} \mathrm{~d} x, \quad u \in H(\Theta)
$$

and the minimization problem

$$
\beta(\Theta)=\inf \{K(u) \mid u \in N(\Theta)\},
$$

where $N(\Theta)=\left\{u \in H(\Theta) \backslash\{0\} \mid\left\langle K^{\prime}(u), u\right\rangle=0\right\}$. Now we prove that problem (2.9) has a positive solution $w_{0}$ such that $K\left(w_{0}\right)=\beta(\Theta)>0$.

Lemma 2.5 For any $u \in H(\Theta) \backslash\{0\}$, there exists a unique $t(u)>0$ such that $t(u) u \in N(\Theta)$. The maximum of $K(t u)$ for $t \geq 0$ is reached at $t=t(u)$, the map

$$
t: H(\Theta) \backslash\{0\} \rightarrow(0,+\infty) ; \quad u \mapsto t(u)
$$

is continuous and the induced continuous map $u \rightarrow t(u) u$ defines a homeomorphism of the unit sphere of $H(\Theta)$ with $N(\Theta)$.

Proof For any given $u \in H(\Theta) \backslash\{0\}$, consider the function $g(t)=K(t u), t \geq 0$. Clearly,

$$
g^{\prime}(t)=0 \quad \Leftrightarrow \quad t u \in N(\Theta) \quad \Leftrightarrow \quad\|u\|^{2}=t^{p-1} \int_{\Omega} h(x)|u|^{p+1} \mathrm{~d} x
$$

It is easy to verify that $g(0)=0, g(t)>0$ for $t>0$ small and $g(t)<0$ for $t>0$ large. Hence, $\max _{t \geq 0} g(t)$ is reached at a unique $t=t(u)$ such that $g^{\prime}(t(u))=0$ and $t(u) u \in N(\Theta)$. To prove the continuity of $t(u)$, assume that $u_{n} \rightarrow u$ in $H(\Theta) \backslash\{0\}$. It is easy to verify that $\left\{t\left(u_{n}\right)\right\}$ is bounded. If a subsequence of $\left\{t\left(u_{n}\right)\right\}$ converges to $t_{0}$, it follows from (2.10) that $t_{0}=t(u)$ and then $t\left(u_{n}\right) \rightarrow t(u)$. Finally the continuous map from the unit sphere of $H(\Theta)$ to $N(\Theta)$, $u \rightarrow t(u) u$, is inverse to the retraction $u \rightarrow \frac{u}{\|u\|}$.

Define

$$
c_{*}=\inf _{u \in H(\Theta) \backslash\{0\}} \max _{t \geq 0} K(t u), \quad c=\inf _{\gamma \in \Gamma} \max _{t \in[0,1]} K(\gamma(t)),
$$

where $\Gamma=\{\gamma \in C([0,1], H(\Theta)) \mid \gamma(0)=0, K(\gamma(1))<0\}$.

Lemma 2.6 $\beta(\Theta)=c_{*}=c>0$ is a critical value of $K$.

Proof From Lemma 2.5, we know that $\beta(\Theta)=c_{*}$. Since $K(t u)<0$ for $u \in H(\Theta) \backslash\{0\}$ and $t$ large, we obtain $c \leq c_{*}$. The manifold $N(\Theta)$ separates $H(\Theta)$ into two components. The component containing the origin also contains a small ball around the origin. Moreover, 
$K(u) \geq 0$ for all $u$ in this component, because $\left\langle K^{\prime}(t u), u\right\rangle \geq 0, \forall t \in[0, t(u)]$. Then each $\gamma \in \Gamma$ has to cross $N(\Theta)$ and $\beta(\Theta) \leq c$. Since the embedding $H(\Theta) \hookrightarrow L^{p+1}(\Theta)$ is compact (see [14]), it is easy to prove that $c>0$ is a critical value of $K$ and $w_{0}$ a positive solution corresponding to $c$.

With the help of Lemma 2.6, we have the following result.

Lemma 2.7 (i) For $\lambda \in\left(0, \lambda_{*}\right)$, there exists $t_{\lambda}>0$ such that

$$
\alpha_{\lambda}(\Omega) \leq \alpha_{\lambda}^{+}(\Omega)<-\frac{1-q}{q+1} t_{\lambda}^{2} \beta_{\lambda}(\Theta)<0
$$

(ii) $J_{\lambda}$ is coercive and bounded below on $M_{\lambda}(\Omega)$ for all $\lambda>0$.

Proof (i) Let $w_{0}$ be a positive solution of problem (2.9) such that $K\left(w_{0}\right)=\beta(\Theta)$. Then

$$
\int_{\Omega} f(x) w_{0}^{q+1} \mathrm{~d} x=\int_{\Theta} f(x) w_{0}^{q+1} \mathrm{~d} x>0 .
$$

Set $t_{\lambda}=t^{+}\left(w_{0}\right)$ as defined by Lemma 2.4(iv). Hence, $t_{\lambda} w_{0} \in M_{\lambda}^{+}(\Omega)$ and

$$
\begin{aligned}
J_{\lambda}\left(t_{\lambda} w_{0}\right) & =\frac{1}{2}\left\|t_{\lambda} w_{0}\right\|^{2}-\frac{\lambda}{q+1} \int_{\Omega} f(x)\left|t_{\lambda} w_{0}\right|^{q+1} \mathrm{~d} x-\frac{1}{p+1} \int_{\Omega} h(x)\left|t_{\lambda} w_{0}\right|^{p+1} \mathrm{~d} x \\
& =\left(\frac{1}{2}-\frac{1}{q+1}\right)\left\|t_{\lambda} w_{0}\right\|^{2}+\left(\frac{1}{q+1}-\frac{1}{p+1}\right) \int_{\Omega} h(x)\left|t_{\lambda} w_{0}\right|^{p+1} \mathrm{~d} x \\
& <-\frac{1-q}{q+1} t_{\lambda}^{2} \beta(\Theta)<0 .
\end{aligned}
$$

This implies

$$
\alpha_{\lambda}(\Omega) \leq \alpha_{\lambda}^{+}(\Omega)<-\frac{1-q}{q+1} t_{\lambda}^{2} \beta(\Theta)<0 .
$$

(ii) For $u \in M_{\lambda}(\Omega)$, we have $\|u\|^{2}=\lambda \int_{\Omega} f(x)|u|^{q+1} \mathrm{~d} x+\int_{\Omega} h(x)|u|^{p+1} \mathrm{~d} x$. Then by the Hölder, Sobolev, and Young inequalities,

$$
\begin{aligned}
J_{\lambda}(u) & =\frac{p-1}{2(p+1)}\|u\|^{2}-\frac{\lambda(p-q)}{(p+1)(q+1)} \int_{\Omega} f(x)|u|^{q+1} \mathrm{~d} x \\
& \geq \frac{p-1}{2(p+1)}\|u\|^{2}-\frac{\lambda(p-q)}{(p+1)(q+1)}|f|_{p^{*}} S^{q+1}\|u\|^{q+1} \\
& \geq \frac{p-1}{4(p+1)}\|u\|^{2}-\lambda^{\frac{2}{1-q}} C(p, q)\left(|f|_{p^{*}} S^{q+1}\right)^{\frac{2}{1-q}}
\end{aligned}
$$

here $C(p, q)=\left[\frac{p-q}{(p+1)(q+1)}\right]^{\frac{2}{1-q}} \cdot\left[\frac{4(p+1)}{p-1}\right]^{\frac{1+q}{1+q}}$.

Thus, $J_{\lambda}$ is coercive on $M_{\lambda}(\Omega)$ and

$$
J_{\lambda}(u) \geq-\lambda^{\frac{2}{1-q}} C(p, q)\left(|f|_{p^{*}} S^{q+1}\right)^{\frac{2}{1-q}}
$$

for all $\lambda>0$. 
Next, we will use the idea of Tarantello [16] to get the following results.

Lemma 2.8 For $\lambda \in\left(0, \lambda_{*}\right)$ and any given $u \in M_{\lambda}(\Omega)$, there exist $\epsilon>0$ and a differentiable functional $\xi: B(0 ; \epsilon) \subset H(\Omega) \rightarrow \mathbb{R}^{+}$such that $\xi(0)=1$, the function $\xi(v)(u+v) \in M_{\lambda}(\Omega)$ and

$$
\left\langle\xi^{\prime}(0), v\right\rangle=\frac{2 \int_{\Omega} \Delta u \Delta v-\lambda(q+1) \int_{\Omega} f|u|^{q-1} u v-(p+1) \int_{\Omega} h|u|^{p-1} u v}{(1-q)\|u\|^{2}-(p-q) \int_{\Omega} h(x)|u|^{p+1} \mathrm{~d} x}
$$

for all $v \in H(\Omega)$.

Proof Define $F: \mathbb{R} \times H(\Omega) \rightarrow \mathbb{R}$ as follows:

$$
F(\xi, w)=\xi^{2}\|u+w\|^{2}-\lambda \xi^{q+1} \int_{\Omega} f(x)|u+w|^{q+1} \mathrm{~d} x-\xi^{p+1} \int_{\Omega} h(x)|u+w|^{p+1} \mathrm{~d} x .
$$

Since $F(1,0)=\left\langle J_{\lambda}^{\prime}(u), u\right\rangle=0$ and by Lemma 2.1, we obtain

$$
\begin{aligned}
F_{\xi}^{\prime}(1,0) & =2\|u\|^{2}-\lambda(q+1) \int_{\Omega} f(x)|u|^{q+1} \mathrm{~d} x-(p+1) \int_{\Omega} h(x)|u|^{p+1} \mathrm{~d} x \\
& =\left\langle\psi_{\lambda}^{\prime}(u), u\right\rangle \neq 0,
\end{aligned}
$$

we can get the desired results applying the implicit function theorem at the point $(1,0)$.

Lemma 2.9 For $\lambda \in\left(0, \lambda_{*}\right)$ and any given $u \in M_{\lambda}^{-}(\Omega)$, there exist $\epsilon>0$ and a differentiable functional $\xi^{-}: B(0 ; \epsilon) \subset H(\Omega) \rightarrow \mathbb{R}^{+}$such that $\xi^{-}(0)=1$, the function $\xi^{-}(v)(u+v) \in M_{\lambda}^{-}(\Omega)$ and

$$
\left\langle\left(\xi^{-}\right)^{\prime}(0), v\right\rangle=\frac{2 \int_{\Omega} \Delta u \Delta v-\lambda(q+1) \int_{\Omega} f|u|^{q-1} u v-(p+1) \int_{\Omega} h|u|^{p-1} u v}{(1-q)\|u\|^{2}-(p-q) \int_{\Omega} h(x)|u|^{p+1} \mathrm{~d} x}
$$

for all $v \in H(\Omega)$.

Proof In view of Lemma 2.8, there exist $\epsilon>0$ and a differentiable functional $\xi^{-}$such that $\xi^{-}(0)=1, \xi^{-}(v)(u+v) \in M_{\lambda}(\Omega)$ for all $v \in B(0 ; \epsilon) \subset H(\Omega)$ and we have (2.12). By use of $u \in M_{\lambda}^{-}(\Omega)$, we have $\left\langle\psi_{\lambda}^{\prime}(u), u\right\rangle<0$. In combination with the continuity of the functions $\psi_{\lambda}^{\prime}$ and $\xi^{-}$, we get $\left\langle\psi_{\lambda}^{\prime}\left(\xi^{-}(v)(u+v)\right), \xi^{-}(v)(u+v)\right\rangle<0$ as $\epsilon$ sufficiently small, this implies that $\xi^{-}(v)(u+v) \in M_{\lambda}^{-}(\Omega)$.

\section{Proof of Theorem 1.1}

Firstly, we provide the existence of minimizing sequences for $J_{\lambda}$ on $M_{\lambda}(\Omega)$ and $M_{\lambda}^{-}(\Omega)$ as $\lambda$ is sufficiently small.

Proposition 3.1 Let $\lambda \in\left(0, \lambda_{*}\right)$, then

(i) there exists a minimizing sequence $\left\{u_{n}\right\} \subset M_{\lambda}(\Omega)$ such that

$$
J_{\lambda}\left(u_{n}\right)=\alpha_{\lambda}(\Omega)+o(1) \quad \text { and } \quad J_{\lambda}^{\prime}\left(u_{n}\right)=o(1) \quad \text { in }[H(\Omega)]^{*} ;
$$


(ii) there exists a minimizing sequence $\left\{u_{n}\right\} \subset M_{\lambda}^{-}(\Omega)$ such that

$$
J_{\lambda}\left(u_{n}\right)=\alpha_{\lambda}^{-}(\Omega)+o(1) \text { and } J_{\lambda}^{\prime}\left(u_{n}\right)=o(1) \quad \text { in }[H(\Omega)]^{*} \text {. }
$$

Proof (i) By Lemma 2.7(ii) and the Ekeland variational principle [17], there exists a minimizing sequence $\left\{u_{n}\right\} \subset M_{\lambda}(\Omega)$ such that

$$
J_{\lambda}\left(u_{n}\right)<\alpha_{\lambda}(\Omega)+\frac{1}{n}
$$

and

$$
J_{\lambda}\left(u_{n}\right)<J_{\lambda}(w)+\frac{1}{n}\left\|w-u_{n}\right\| \quad \text { for each } w \in M_{\lambda}(\Omega) .
$$

Taking $n$ large, from Lemma 2.7(i) and (3.1), we have

$$
\begin{aligned}
J_{\lambda}\left(u_{n}\right) & =\left(\frac{1}{2}-\frac{1}{p+1}\right)\left\|u_{n}\right\|^{2}-\left(\frac{1}{q+1}-\frac{1}{p+1}\right) \lambda \int_{\Omega} f(x)\left|u_{n}\right|^{q+1} \mathrm{~d} x \\
& <\alpha_{\lambda}(\Omega)+\frac{1}{n}<-\frac{1-q}{q+1} t_{\lambda}^{2} \beta(\Theta) .
\end{aligned}
$$

This implies

$$
|f|_{p^{*}} S^{q+1}\left\|u_{n}\right\|^{q+1} \geq \int_{\Omega} f(x)\left|u_{n}\right|^{q+1} \mathrm{~d} x>\frac{(p+1)(1-q)}{\lambda(p-q)} t_{\lambda}^{2} \beta(\Theta)>0,
$$

that is

$$
\left\|u_{n}\right\|>\left[\frac{(p+1)(1-q)}{\lambda(p-q)} t_{\lambda}^{2} \beta(\Theta) S^{-(q+1)}|f|_{p^{*}}^{-1}\right]^{\frac{1}{q+1}}
$$

Now, we will show that

$$
\left\langle J_{\lambda}^{\prime}\left(u_{n}\right), \varphi\right\rangle \rightarrow 0 \quad \text { as } n \rightarrow \infty, \forall \varphi \in H(\Omega)
$$

Exactly as in Lemma 2.8 we may apply suitable functionals $\xi_{n}(v)>0$ to $u_{n}$ and obtain

$$
\xi_{n}(v)\left(u_{n}+v\right) \in M_{\lambda}(\Omega), \quad \forall v \in H(\Omega),\|v\|<\epsilon_{n} .
$$

Hence, if $\varphi \in H(\Omega)$ and $s>0$ small, substituting in (3.6) $v=s \varphi$ and applying (3.2), we have

$$
\begin{aligned}
& \frac{1}{n}\left[\left|\xi_{n}(s \varphi)-1\right| \cdot\left\|u_{n}\right\|+\xi_{n}(s \varphi)\|s \varphi\|\right] \\
& \geq J_{\lambda}\left(u_{n}\right)-J_{\lambda}\left(\xi_{n}(s \varphi)\left(u_{n}+s \varphi\right)\right) \\
& =\frac{1}{2}\left\|u_{n}\right\|^{2}-\frac{\lambda}{q+1} \int_{\Omega} f(x)\left|u_{n}\right|^{q+1} \mathrm{~d} x-\frac{1}{p+1} \int_{\Omega} h(x)\left|u_{n}\right|^{p+1} \mathrm{~d} x \\
& \quad-\frac{1}{2} \xi_{n}^{2}(s \varphi)\left\|u_{n}+s \varphi\right\|^{2}+\frac{\lambda}{q+1} \xi_{n}^{q+1}(s \varphi) \int_{\Omega} f(x)\left|u_{n}+s \varphi\right|^{q+1} \mathrm{~d} x
\end{aligned}
$$




$$
\begin{aligned}
& +\frac{1}{p+1} \xi_{n}^{p+1}(s \varphi) \int_{\Omega} h(x)\left|u_{n}+s \varphi\right|^{p+1} \mathrm{~d} x \\
= & -\frac{\xi_{n}^{2}(s \varphi)-1}{2}\left\|u_{n}+s \varphi\right\|^{2}-\frac{1}{2}\left(\left\|u_{n}+s \varphi\right\|^{2}-\left\|u_{n}\right\|^{2}\right) \\
& +\lambda \frac{\xi_{n}^{q+1}(s \varphi)-1}{q+1} \int_{\Omega} f(x)\left|u_{n}+s \varphi\right|^{q+1} \mathrm{~d} x \\
& +\frac{\lambda}{q+1} \int_{\Omega} f(x)\left(\left|u_{n}+s \varphi\right|^{q+1}-\left|u_{n}\right|^{q+1}\right) \mathrm{d} x \\
& +\frac{\xi_{n}^{p+1}(s \varphi)-1}{p+1} \int_{\Omega} h(x)\left|u_{n}+s \varphi\right|^{p+1} \mathrm{~d} x+\frac{1}{p+1} \int_{\Omega} h(x)\left(\left|u_{n}+s \varphi\right|^{p+1}-\left|u_{n}\right|^{p+1}\right) \mathrm{d} x .
\end{aligned}
$$

Dividing by $s>0$ and passing to the limit as $s \rightarrow 0$ we derive

$$
\begin{aligned}
\frac{1}{n}\left[\left|\xi_{n}^{\prime}(0) \varphi\right|\left\|u_{n}\right\|+\|\varphi\|\right] \\
\geq-\left[\xi_{n}^{\prime}(0) \varphi\right]\left[\left\|u_{n}\right\|^{2}-\lambda \int_{\Omega} f(x)\left|u_{n}\right|^{q+1} \mathrm{~d} x-\int_{\Omega} h(x)\left|u_{n}\right|^{p+1} \mathrm{~d} x\right] \\
\quad-\int_{\Omega} \Delta u_{n} \Delta \varphi \mathrm{d} x+\lambda \int_{\Omega} f(x)\left|u_{n}\right|^{q-1} u_{n} \varphi \mathrm{d} x+\int_{\Omega} h(x)\left|u_{n}\right|^{p-1} u_{n} \varphi \mathrm{d} x \\
=-\int_{\Omega} \Delta u_{n} \Delta \varphi \mathrm{d} x+\lambda \int_{\Omega} f(x)\left|u_{n}\right|^{q-1} u_{n} \varphi \mathrm{d} x+\int_{\Omega} h(x)\left|u_{n}\right|^{p-1} u_{n} \varphi \mathrm{d} x .
\end{aligned}
$$

Since

$$
\xi_{n}^{\prime}(0) \varphi=\frac{2 \int_{\Omega} \Delta u_{n} \Delta \varphi-\lambda(q+1) \int_{\Omega} f\left|u_{n}\right|^{q-1} u_{n} \varphi-(p+1) \int_{\Omega} h\left|u_{n}\right|^{p-1} u_{n} \varphi}{(1-q)\left\|u_{n}\right\|^{2}-(p-q) \int_{\Omega} h(x)\left|u_{n}\right|^{p+1} \mathrm{~d} x},
$$

by the boundedness of $u_{n}$ we get

$$
\left\|\xi_{n}^{\prime}(0)\right\| \leq \frac{C_{1}}{\left.\left|(1-q)\left\|u_{n}\right\|^{2}-(p-q) \int_{\Omega} h(x)\right| u_{n}\right|^{p+1} \mathrm{~d} x \mid}
$$

for a suitable positive constant $C_{1}$.

Next, we show that $\left.\left|(1-q)\left\|u_{n}\right\|^{2}-(p-q) \int_{\Omega} h(x)\right| u_{n}\right|^{p+1} \mathrm{~d} x \mid$ is bounded away from zero. Arguing by contradiction, assume that

$$
(1-q)\left\|u_{n}\right\|^{2}-(p-q) \int_{\Omega} h(x)\left|u_{n}\right|^{p+1} \mathrm{~d} x=o(1), \quad n \rightarrow \infty .
$$

Since $u_{n} \in M_{\lambda}(\Omega)$, we have

$$
\left\|u_{n}\right\|^{2}=\lambda \int_{\Omega} f(x)\left|u_{n}\right|^{q+1} \mathrm{~d} x+\int_{\Omega} h(x)\left|u_{n}\right|^{p+1} \mathrm{~d} x,
$$

and consequently by (3.9),

$$
\frac{p-1}{1-q} \int_{\Omega} h(x)\left|u_{n}\right|^{p+1} \mathrm{~d} x=\lambda \int_{\Omega} f(x)\left|u_{n}\right|^{q+1} \mathrm{~d} x+o(1), \quad n \rightarrow \infty .
$$


Then by (3.4), the Hölder inequality, Sobolev inequality and (3.9)-(3.10), we obtain

$$
\begin{aligned}
0 & <\left(\lambda_{*}-\lambda\right) \int_{\Omega} f(x)\left|u_{n}\right|^{q+1} \mathrm{~d} x \\
& \leq \frac{p-1}{1-q} \int_{\Omega} h(x)\left|u_{n}\right|^{p+1} \mathrm{~d} x\left[\frac{(p-q) \int_{\Omega} h(x)\left|u_{n}\right|^{p+1} \mathrm{~d} x}{(1-q)\left\|u_{n}\right\|^{2}}\right]^{\frac{q-p}{p-1}}-\lambda \int_{\Omega} f(x)\left|u_{n}\right|^{q+1} \mathrm{~d} x \\
& =o(1),
\end{aligned}
$$

moreover, $\left\|u_{n}\right\|=o(1)$, which contradicts (3.5).

Thus, we get from (3.8) that

$$
\left\|\xi_{n}^{\prime}(0)\right\| \leq C_{2}, \quad \text { independent of } n
$$

Hence, by (3.7) it follows that

$$
\int_{\Omega} \Delta u_{n} \Delta \varphi \mathrm{d} x-\lambda \int_{\Omega} f(x)\left|u_{n}\right|^{q-1} u_{n} \varphi \mathrm{d} x-\int_{\Omega} h(x)\left|u_{n}\right|^{p-1} u_{n} \varphi \mathrm{d} x \geq-\frac{C_{3}}{n}
$$

which implies that $\left\langle J_{\lambda}^{\prime}\left(u_{n}\right), \varphi\right\rangle \rightarrow 0$, as $n \rightarrow \infty$.

(ii) Similar to the arguments in (i), by Lemma 2.9 and Lemma 2.2, we can prove (ii).

Now, we establish the existence of a local minimum for $J_{\lambda}$ on $M_{\lambda}^{+}(\Omega)$.

Theorem 3.1 Let $\lambda \in\left(0, \lambda_{*}\right)$, then the functional $J_{\lambda}$ has a minimizer $u_{0}^{+}$in $M_{\lambda}^{+}(\Omega)$ and it satisfies

(i) $J_{\lambda}\left(u_{0}^{+}\right)=\alpha_{\lambda}(\Omega)=\alpha_{\lambda}^{+}(\Omega)$;

(ii) $u_{0}^{+}$is a positive solution of problem (1.3);

(iii) $J_{\lambda}\left(u_{0}^{+}\right) \rightarrow 0$ as $\lambda \rightarrow 0$.

Proof By Proposition 3.1(i), there is a minimizing sequence $\left\{u_{n}\right\}$ for $J_{\lambda}$ on $M_{\lambda}(\Omega)$ such that

$$
J_{\lambda}\left(u_{n}\right)=\alpha_{\lambda}(\Omega)+o(1) \text { and } J_{\lambda}^{\prime}\left(u_{n}\right)=o(1) \quad \text { in }[H(\Omega)]^{*} .
$$

Then by Lemma 2.7 and the compact imbedding theorem, there exist a subsequence $\left\{u_{n}\right\}$ and $u_{0}^{+} \in H(\Omega)$ such that

$$
\begin{aligned}
& u_{n} \rightarrow u_{0}^{+} \quad \text { weakly in } H(\Omega), \\
& u_{n} \rightarrow u_{0}^{+} \quad \text { strongly in } L^{p+1}(\Omega)
\end{aligned}
$$

and

$$
u_{n} \rightarrow u_{0}^{+} \quad \text { strongly in } L^{q+1}(\Omega) .
$$

First, we claim that

$$
\int_{\Omega} f(x)\left|u_{0}^{+}\right|^{q+1} \mathrm{~d} x>0
$$


If not, by (3.14) we conclude that

$$
\int_{\Omega} f(x)\left|u_{n}\right|^{q+1} \mathrm{~d} x \rightarrow \int_{\Omega} f(x)\left|u_{0}^{+}\right|^{q+1} \mathrm{~d} x \leq 0 \quad \text { as } n \rightarrow \infty .
$$

Therefore, as $n \rightarrow \infty$,

$$
\begin{aligned}
J_{\lambda}\left(u_{n}\right) & =\frac{1}{2}\left\|u_{n}\right\|^{2}-\frac{\lambda}{q+1} \int_{\Omega} f(x)\left|u_{n}\right|^{q+1} \mathrm{~d} x-\frac{1}{p+1} \int_{\Omega} h(x)\left|u_{n}\right|^{p+1} \mathrm{~d} x \\
& =\left(\frac{1}{2}-\frac{1}{q+1}\right) \lambda \int_{\Omega} f(x)\left|u_{n}\right|^{q+1} \mathrm{~d} x+\left(\frac{1}{2}-\frac{1}{p+1}\right) \int_{\Omega} h(x)\left|u_{n}\right|^{p+1} \mathrm{~d} x \\
& =\left(\frac{1}{2}-\frac{1}{q+1}\right) \lambda \int_{\Omega} f(x)\left|u_{0}^{+}\right|^{q+1} \mathrm{~d} x+\left(\frac{1}{2}-\frac{1}{p+1}\right) \int_{\Omega} h(x)\left|u_{0}^{+}\right|^{p+1} \mathrm{~d} x+o(1),
\end{aligned}
$$

this contradicts $J_{\lambda}\left(u_{n}\right) \rightarrow \alpha_{\lambda}(\Omega)<0$ as $n \rightarrow \infty$.

In combination with (3.11)-(3.14), it is easy to verify that $u_{0}^{+} \in M_{\lambda}(\Omega)$ is a nontrivial weak solution of problem (1.3).

Now we prove that $u_{n} \rightarrow u_{0}^{+}$strongly in $H(\Omega)$. Supposing the contrary, then $\left\|u_{0}^{+}\right\|<$ $\liminf _{n \rightarrow \infty}\left\|u_{n}\right\|$ and so

$$
\begin{aligned}
& \left\|u_{0}^{+}\right\|^{2}-\lambda \int_{\Omega} f(x)\left|u_{0}^{+}\right|^{q+1} \mathrm{~d} x-\int_{\Omega} h(x)\left|u_{0}^{+}\right|^{p+1} \mathrm{~d} x \\
& \quad<\liminf _{n \rightarrow \infty}\left(\left\|u_{n}\right\|^{2}-\lambda \int_{\Omega} f(x)\left|u_{n}\right|^{q+1} \mathrm{~d} x-\int_{\Omega} h(x)\left|u_{n}\right|^{p+1} \mathrm{~d} x\right)=0,
\end{aligned}
$$

this contradicts $u_{0}^{+} \in M_{\lambda}(\Omega)$. Hence, $u_{n} \rightarrow u_{0}^{+}$strongly in $H(\Omega)$. This implies

$$
J_{\lambda}\left(u_{n}\right) \rightarrow J_{\lambda}\left(u_{0}^{+}\right)=\alpha_{\lambda}(\Omega) \quad \text { as } n \rightarrow \infty
$$

Moreover, we have $u_{0}^{+} \in M_{\lambda}^{+}(\Omega)$. In fact, if $u_{0}^{+} \in M_{\lambda}^{-}(\Omega)$, by Lemma 2.4 , there exist unique $t_{0}^{+}$and $t_{0}^{-}$such that $t_{0}^{+} u_{0}^{+} \in M_{\lambda}^{+}(\Omega)$ and $t_{0}^{-} u_{0}^{+} \in M_{\lambda}^{-}(\Omega)$, we get $t_{0}^{+}<t_{0}^{-}=1$. Since

$$
\frac{\mathrm{d} J_{\lambda}(t u)}{\mathrm{d} t}=0 \text { if and only if } t=t_{0}^{+} \text {and } t_{0}^{-}
$$

and

$$
\left.\frac{\mathrm{d}^{2} J_{\lambda}(t u)}{\mathrm{d} t^{2}}\right|_{t=t_{0}^{+}}>0,\left.\quad \frac{\mathrm{d}^{2} J_{\lambda}(t u)}{\mathrm{d} t^{2}}\right|_{t=t_{0}^{-}}<0,
$$

there exists $\tilde{t} \in\left(t_{0}^{+}, t_{0}^{-}\right]$such that $J_{\lambda}\left(t_{0}^{+} u_{0}^{+}\right)<J_{\lambda}\left(\tilde{t} u_{0}^{+}\right)$. By Lemma 2.4,

$$
J_{\lambda}\left(t_{0}^{+} u_{0}^{+}\right)<J_{\lambda}\left(\tilde{t} u_{0}^{+}\right) \leq J_{\lambda}\left(t_{0}^{-} u_{0}^{+}\right)=J_{\lambda}\left(u_{0}^{+}\right)
$$

which is a contradiction. Since $J_{\lambda}\left(u_{0}^{+}\right)=J_{\lambda}\left(\left|u_{0}^{+}\right|\right)$and $\left|u_{0}^{+}\right| \in M_{\lambda}^{+}(\Omega)$, by Lemma 2.3 we may assume that $u_{0}^{+}$is a nonnegative weak solution to problem (1.3). Applying the regularity theory and strong maximum principle of elliptic equations, we find that $u_{0}^{+}$is one positive solution of problem (1.3). In addition, by Lemma 2.7,

$$
0>J_{\lambda}\left(u_{0}^{+}\right) \geq-\lambda^{\frac{2}{1-q}} C(p, q)\left(|f|_{p^{*}} S^{q+1}\right)^{\frac{2}{1-q}}
$$

which implies that $J_{\lambda}\left(u_{0}^{+}\right) \rightarrow 0$ as $\lambda \rightarrow 0$. 
Next, we establish the existence of a local minimum for $J_{\lambda}$ on $M_{\lambda}^{-}(\Omega)$.

Theorem 3.2 Let $\lambda \in\left(0, \lambda_{*}\right)$, then the functional $J_{\lambda}$ has a minimizer $u_{0}^{-}$in $M_{\lambda}^{-}(\Omega)$ and it satisfies

(i) $J_{\lambda}\left(u_{0}^{-}\right)=\alpha_{\lambda}^{-}(\Omega)$;

(ii) $u_{0}^{-}$is a positive solution of problem (1.3).

Proof By Proposition 3.1(ii), there is a minimizing sequence $\left\{u_{n}\right\}$ for $J_{\lambda}$ on $M_{\lambda}^{-}(\Omega)$ such that

$$
J_{\lambda}\left(u_{n}\right)=\alpha_{\lambda}^{-}(\Omega)+o(1) \quad \text { and } \quad J_{\lambda}^{\prime}\left(u_{n}\right)=o(1) \quad \text { in }[H(\Omega)]^{*} .
$$

Then by Lemma 2.7 and the compact imbedding theorem, there exist a subsequence $\left\{u_{n}\right\}$ and $u_{0}^{-} \in H(\Omega)$ such that

$$
\begin{array}{ll}
u_{n} \rightarrow u_{0}^{-} & \text {weakly in } H(\Omega), \\
u_{n} \rightarrow u_{0}^{-} & \text {strongly in } L^{p+1}(\Omega)
\end{array}
$$

and

$$
u_{n} \rightarrow u_{0}^{-} \quad \text { strongly in } L^{q+1}(\Omega) .
$$

Connecting with Lemma 2.2, it is easy to see that $u_{0}^{-} \in M_{\lambda}(\Omega)$ is a nontrivial weak solution of problem (1.3).

Next we prove that $u_{n} \rightarrow u_{0}^{-}$strongly in $H(\Omega)$. Supposing the contrary, then $\left\|u_{0}^{-}\right\|<$ $\liminf _{n \rightarrow \infty}\left\|u_{n}\right\|$ and so

$$
\begin{aligned}
& \left\|u_{0}^{-}\right\|^{2}-\lambda \int_{\Omega} f(x)\left|u_{0}^{-}\right|^{q+1} \mathrm{~d} x-\int_{\Omega} h(x)\left|u_{0}^{-}\right|^{p+1} \mathrm{~d} x \\
& \quad<\liminf _{n \rightarrow \infty}\left(\left\|u_{n}\right\|^{2}-\lambda \int_{\Omega} f(x)\left|u_{n}\right|^{q+1} \mathrm{~d} x-\int_{\Omega} h(x)\left|u_{n}\right|^{p+1} \mathrm{~d} x\right)=0,
\end{aligned}
$$

this contradicts $u_{0}^{-} \in M_{\lambda}(\Omega)$. Hence, $u_{n} \rightarrow u_{0}^{-}$strongly in $H(\Omega)$. This implies

$$
J_{\lambda}\left(u_{n}\right) \rightarrow J_{\lambda}\left(u_{0}^{-}\right)=\alpha_{\lambda}^{-}(\Omega) \quad \text { as } n \rightarrow \infty .
$$

In addition, from Lemma 2.4(ii)-(iii), we have $u_{0}^{-} \in M_{\lambda}^{-}(\Omega)$. Since $J_{\lambda}\left(u_{0}^{-}\right)=J_{\lambda}\left(\left|u_{0}^{-}\right|\right)$and $\left|u_{0}^{-}\right| \in M_{\lambda}^{-}(\Omega)$, by Lemma 2.3 we may assume that $u_{0}^{-}$is a nonnegative weak solution to problem (1.3). Applying the regularity theory and strong maximum principle of elliptic equations, we see that $u_{0}^{-}$is one positive solution of problem (1.3).

Proof of Theorem 1.1 It is an immediate consequence of Theorems 3.1 and 3.2. 
Authors' contributions

The authors read and approved the final manuscript.

\section{Author details}

'School of Mathematics and Statistics, Lanzhou University, Lanzhou, Gansu 730000, P.R. China. ${ }^{2}$ Key Laboratory of Applied Mathematics and Complex Systems, Lanzhou University, Lanzhou, Gansu 730000, P.R. China. ${ }^{3}$ College of Mathematics and Statistics, Northwest Normal University, Lanzhou, 730070, P.R. China.

\section{Acknowledgements}

This work is partly supported by NNSF $(11101404,11201204,11361053)$ of China and the Young Teachers Scientific Research Ability Promotion Plan of Northwest Normal University (NWNU-LKQN-11-5).

Received: 1 March 2014 Accepted: 29 April 2014 Published: 14 May 2014

\section{References}

1. Alama, S, Tarantello, G: On semilinear elliptic equations with indefinite nonlinearities. Calc. Var. Partial Differ. Equ. 1, 439-475 (1993)

2. Ambrosetti, A, Brezis, H, Cerami, G: Combined effects of concave and convex nonlinearities in some elliptic problems. J. Funct. Anal. 122, 519-543 (1994)

3. Bartsch, T, Willem, M: On an elliptic equation with concave and convex nonlinearities. Proc. Am. Math. Soc. 123, 3555-3561 (1995)

4. Brown, KJ, Zhang, Y: The Nehari manifold for a semilinear elliptic equation with a sign-changing weight function. J. Differ. Equ. 193, 481-499 (2003)

5. Li, S, Wu, S, Zhou, HS: Solutions to semilinear elliptic problems with combined nonlinearities. J. Differ. Equ. 185 200-224 (2001)

6. Sun, Y, Li, S: A nonlinear elliptic equation with critical exponent: estimates for extremal values. Nonlinear Anal. 69 1856-1869 (2008)

7. Tang, M: Exact multiplicity for semilinear elliptic Dirichlet problems involving concave and convex nonlinearities. Proc. R. Soc. Edinb., Sect. A 133, 705-717 (2003)

8. Wu, TF: On semilinear elliptic equations involving concave-convex nonlinearities and sign-changing weight function. J. Math. Anal. Appl. 318, 253-270 (2006)

9. Guo, D, Lakshmikantham, V: Nonlinear Problems in Abstract Cones. Academic Press, New York (1988)

10. Willem, M: Minimax Theorems. Birkhäuser, Basel (1996)

11. Ebobisse, F, Ahmedou, MO: On a nonlinear fourth-order elliptic equation involving the critical Sobolev exponent. Nonlinear Anal. 52, 1535-1552 (2003)

12. Gazzola, F, Grunau, H-C, Squassina, M: Existence and nonexistence results for critical growth biharmonic elliptic equations. Calc. Var. Partial Differ. Equ. 18, 117-143 (2003)

13. Zhang, Y: Positive solutions of semilinear biharmonic equations with critical Sobolev exponents. Nonlinear Anal. 75 , 55-67 (2012)

14. Adams, RA: Sobolev Spaces. Academic Press, New York (1975)

15. Rabinowitz, PH: Minimax Methods in Critical Point Theory with Applications to Differential Equations. Reg. Conf. Ser Math. Am. Math. Soc., Providence (1986)

16. Tarantello, G: On nonhomogeneous elliptic involving critical Sobolev exponent. Ann. Inst. Henri Poincaré, Anal. Non Linéaire 9, 281-304 (1992)

17. Ekeland, I: On the variational principle. J. Math. Anal. Appl. 17, 324-353 (1974)

10.1186/1687-2770-2014-117

Cite this article as: Yang and Wang: On semilinear biharmonic equations with concave-convex nonlinearities involving weight functions. Boundary Value Problems 2014, 2014:117

\section{Submit your manuscript to a SpringerOpen ${ }^{0}$ journal and benefit from:}

- Convenient online submission

- Rigorous peer review

Immediate publication on acceptance

Open access: articles freely available online

- High visibility within the field

- Retaining the copyright to your article 